\title{
Depressionsbehandlung - noch viele ungelöste Fragen
}

\section{Ulrich Voderholzer}

Schön Klinik Roseneck, Prien am Chiemsee, Deutschland

Der Schwerpunkt der vorliegenden letzten Ausgabe von Verhaltenstherapie in 2016 widmet sich dem Thema «Depression». Depressive Erkrankungen zählen weltweit zu den häufigsten Erkrankungen. Epidemiologische Studien können zwar nicht belegen, dass die Prävalenz depressiver Störungen zugenommen hat. Allerdings hat deren sozioökonomische Bedeutung durch eine massiv gestiegene Rate an Krankschreibungen und Frühberentungen aufgrund psychischer Störungen (insbesondere Depression) stark zugenommen. Relativ zu anderen körperlichen Erkrankungen gesehen, die in früheren Jahrzehnten eine viel stärkere Bedeutung hatten, hat die Gesamtbedeutung depressiver Erkrankungen in modernen Industriegesellschaften und vermutlich auch weltweit erheblich zugenommen. Besorgniserregend ist dabei auch die Tendenz zu einem früheren Erkrankungsbeginn. Dieser zeigt sich nicht nur in der Versorgung mit einer steigenden Zahl ambulanter und stationärer Behandlungen von depressiven Störungen im Jugendalter, sondern auch in epidemiologischen Studien.

Angesichts dieser Situation sei eine kurze Reflexion darüber erlaubt, wo wir heute mit unseren wissenschaftlichen Erkenntnissen bezüglich der Depressionsbehandlung und ihrer Umsetzung in der Praxis denn eigentlichen stehen.

Sicherlich hat der Erkenntniszuwachs sowohl hinsichtlich der Wirksamkeit von somatischen Behandlungsmethoden (Pharmakotherapie, andere somatische Therapieverfahren) als auch bezüglich der Wirksamkeit psychotherapeutischer Verfahren erheblich zugenommen. In diesem Jahr ist bereits die zweite aktualisierte Version der S3-Leitlinie Depression, bei der Fachverbände, Selbsthilfeverbände usw. in sehr großer Zahl mitgewirkt haben, erschienen.

Man fragt sich nur: Warum ist die Gesellschaft angesichts dieses enorm gestiegenen Wissenszuwachses bezüglich depressiver Erkrankungen nicht erfolgreicher in der Bekämpfung von Depressionen? Schließlich hat in anderen medizinischen Bereichen, wie etwa bei den Herz-Kreislauf-Erkrankungen, die Zahl der Frühberentungen deutlich abgenommen, und die Lebenserwartung ist deutlich gestiegen. Warum wirkt sich also der massiv gestiegene Erkenntniszuwachs bei depressiven Erkrankungen nicht auch in einem Rückgang der Frühberentungen und Krankschreibungen aufgrund von Depression aus?

\section{KARGER}

(c) 2016 S. Karger GmbH, Freiburg
Offensichtlich gibt es noch Nachholbedarf beim Transfer des zunehmenden Wissens in die Versorgungspraxis, und wir müssen selbstkritisch eingestehen, dass wir mit unserem Versorgungssystem noch effizienter werden müssen.

Auf der anderen Seite gibt es auch noch viele wichtige, für die Versorgung relevante Fragestellungen, die empirisch noch nicht oder nicht gut untersucht sind. Hier sollen nur einige Beispiele genannt werden, sowohl aus dem Bereich der Therapie- als auch der Versorgungsforschung.

\section{Placebowirkungen in der Akuttherapie}

Wir wissen, dass Placeboeffekte bei depressiven Störungen eine große Rolle spielen [Kirsch, 2016] - bei Medikamenten wie auch bei der Psychotherapie. Das heißt, einem Patienten mit Depression eine Therapie anzubieten und ihm Hoffnung zu vermitteln, ist schon ein starker Wirkfaktor, unabhängig von der Art der Therapie.

Doch was sind dann die eigentlichen Elemente in der Akuttherapie, die einen wirklichen Mehrwert für die Akutbehandlung und für das Langzeit-Outcome darstellen?

Für eine effiziente Anwendung von Akuttherapie ist dies eine wichtige Fragestellung.

\section{Richtlinien-Psychotherapie}

Zwischen dem Erkenntnisfortschritt in der Wirksamkeit von Psychotherapie bei Depression und dem gegenwärtigen System der Richtlinien-Psychotherapie ist mittlerweile eine immer größere Kluft entstanden. Ein Beispiel ist die interpersonelle Psychotherapie, für die es mittlerweile mindestens so viele Wirksamkeitsbelege bei Depression gibt wie für tiefenpsychologische Verfahren, die aber in Deutschland weiterhin im Rahmen der Richtlinien-Psychotherapie nicht anerkannt ist. 


\section{Personalisierte Medizin}

Welche Therapie ist für den einzelnen die beste? Auch wenn dieses Thema derzeit große Aufmerksamkeit erregt und den Gegenstand vieler Forschungsansätze darstellt, wissen wir letztendlich noch nicht viel darüber. Im Bereich der Pharmakotherapie werden z.B. genetische Tests propagiert, mit deren Hilfe Empfehlungen für bestimmte Antidepressiva abgeleitet werden sollen, die beim Betroffenen besser wirken sollen als andere. Bisher liegen aber keine prospektiven kontrollierten Studien vor, die den Vorteil der Gentest-gestützten Entscheidung für eine bestimmte Therapie im Vergleich zur klinischen Entscheidung im Hinblick auf eine bessere Therapie-Response zeigen konnten. Ähnliches gilt auch für die Psychotherapie.

\section{Wirkungen von Therapien auf den Verlauf depressiver Erkrankungen / langfristige Wirkungen von Therapien}

Gerade bei dem häufig chronischen und rezidivierenden Krankheitsbild «Depression» wurde dieser Bereich bisher vernachlässigt und sollte künftig stärker erforscht werden [Voderholzer und Barton, 2016]. Es gibt in der somatischen Medizin viele Beispiele für Therapieansätze, die aufgrund neuer Erkenntnisse zum Langzeitverlauf wieder aufgegeben wurden. So gesehen ist es eine enorm wichtige Fragestellung, ob z.B. Antidepressiva eine günstige oder eventuell auch schädliche Wirkung auf den Langzeitverlauf von Depressionen haben.

\section{Setting-Fragen}

Auch Setting-Fragen sind bislang nicht wirklich empirisch untersucht worden.

Die Behandlung akuter schwerer Depressionen erfolgt in Deutschland ambulant, teilstationär oder stationär. Abgesehen davon, dass die Entscheidung für ein Setting oftmals auch von der
Verfügbarkeit (lange Wartezeiten für einen ersten Termin bei einem Psychiater oder bei einem Psychotherapeuten) abhängt, gibt es so gut wie keine aussagekräftigen Untersuchungen darüber, ob ein Setting einem anderen Setting langfristig bezüglich des Outcome überlegen ist.

\section{Wohnortnahe oder wohnortferne Versorgung}

Vielfach wird propagiert, dass die Versorgung psychischer Erkrankungen wohnortnah erfolgen sollte und damit ambulante, teilstationäre und stationäre Versorgungsmöglichkeiten möglichst in der näheren Umgebung der Betroffenen vorgehalten werden müssen. Allerdings wurde bisher nicht empirisch untersucht, ob damit bessere Behandlungsergebnisse verbunden sind [Diedrich et al., 2016; in diesem Heft]. Soweit uns bekannt ist, liegen auch keine Befragungen dazu vor, ob Betroffene, z.B. im Falle einer notwendigen stationären Behandlung, lieber wohnortnah oder etwas entfernter vom Wohnort therapiert werden wollen.

\section{Wie hilfreich sind Telemedizin und Online-Therapie in der Routineversorgung?}

Weltweit wächst die Zahl von Online-Therapieangeboten, und auch der Beitrag von Steinmann et al. [2016] in diesem Heft zu einem telefongestützten manualisierten verhaltenstherapeutischen Interventionsprogramm für depressive Störungen ist eine Beispiel für innovative neue Ansätze. Die Überprüfung von deren Wirksamkeit in der Routineversorgung muss aber noch abgewartet werden.

Die genannten Beispiele sollten zeigen, dass es viele, für die Praxis sehr relevante Fragestellungen in der Behandlung depressiver Erkrankungen gibt, bei denen wir (noch) nicht ausreichend auf empirische Forschung zurückgreifen können. Viele weitere Fragestellungen ließen sich aufführen. Hier bestehen also noch beträchtliche Herausforderungen, um Depressionen in der Gesellschaft zukünftig effektiver zu bekämpfen.

\section{Literatur}

Diedrich A, Ewald H, Langs G, et al: Stationäre Behandlung depressiver Erkrankungen wohnortnah oder wohnortfern: Gibt es Unterschiede im Therapieergebnis? Verhaltenstherapie 2016;26:doi: 10.1159/000452648.

Kirsch I: Der Placeboeffekt in der antidepressiven Behandlung. Verhaltenstherapie 2016;26:55-61.
Steinmann M, Heddaeus D, Liebherz S, et al: Telefongestützte Verhaltenstherapie als niedrigschwellige Intervention bei Depression: Ein Behandlungsprogramm für den deutschen Sprachraum. Verhaltenstherapie 2016;26:doi: 10.1159/000452435.
Voderholzer U, Barton B: Langfristige Wirkung von Psychotherapie bei nichtchronischen Depressionen: Ein systematisches Review von Studien im Vergleich mit Pharmakotherapie. Verhaltenstherapie 2016;26:108115. 